\title{
Genome-wide analysis of lectin receptor-like kinases family from potato (Solanum tuberosum L.)
}

Weina Zhang ${ }^{1}$, Zhongjian Chen ${ }^{2}$, Yichen Kang ${ }^{1}$, Yanling Fan ${ }^{1}$, Yuhui Liu ${ }^{3}$, Xinyu Yang ${ }^{1}$, Mingfu Shi ${ }^{1}$, Kai Yao Shuhao Qin ${ }^{\text {Corresp. } 1}$

${ }^{1}$ College of Horticulture, Gansu Agricultural University, Lanzhou, China

2 Agro-biological Gene Research Center, Guangdong Academy of Agricultural Sciences, Guangzhou, China

3 Gansu Key Laboratory of Crop Improvement and Germplasm Enhancement, Gansu Agricultural University, Lanzhou, China

Corresponding Author: Shuhao Qin

Email address: qinsh@gsau.edu.cn

Lectin receptor-like kinases (LecRLKs) are involved in response to diverse environmental stresses and pathogenic microbes. A comprehensive acknowledgment of the family members in potato (Solanum tuberosum) genome is largely limited until now. In total, 113 potato LecRLKs (StLecRLKs) were first identified, including 85 G-type, 26 L-type and 2 Ctype members. Based on phylogenetic analysis, StLecRLKs were sub-grouped into seven clades, including C-type, L-type, G-I, G-II, G-III G-IV and G-V. Chromosomal distribution and gene duplication analysis revealed the expansion of StLecRLKs occurred majorly through tandem duplication although the whole-genome duplication (WGD)/segmental duplication events were found. Cis-elements in the StLeCRLKs promoter region responded mainly to signals of defense and stress, phytohormone, biotic or abiotic stress. Moreover, expressional investigations indicated that the family members of the clades L-type, G-I, GIV and G-V were responsive to both bacterial and fungal infection. Based on qRT-PCR analysis, the expressions of PGSC0003DMP400055136 and PGSC0003DMP400067047 were strongly induced in all treatments by both Fusarium sulphureum (Fs) and Phytophthora infestans (Pi) inoculation. The present study provides valuable information for LecRLKs gene family in potato genome, and establishes a foundation for further research into the functional analysis. 


\section{Genome-wide analysis of lectin receptor-like kinases family from}

\section{2 potato (Solanum tuberosum L.)}

3 Weina Zhang ${ }^{1}$, Zhongjian Chen $^{2}$, Yichen Kang ${ }^{1}$, Yanling Fan ${ }^{1}$, Yuhui Liu ${ }^{3}$, Xinyu Yang ${ }^{1}$, Mingfu Shi ${ }^{1}$, Kai Yao ${ }^{1}$,

4 Shuhao Qin ${ }^{1, *}$

$5 \quad{ }^{1}$ College of Horticulture, Gansu Agricultural University, Lanzhou 730070, China;

$6 \quad{ }^{2}$ Agro-biological Gene Research Center, Guangdong Academy of Agricultural Sciences, Guangzhou 510640, China;

$7{ }^{3}$ Gansu Key Laboratory of Crop Improvement and Germplasm Enhancement, Gansu Agricultural University,

8 Lanzhou 730070 , China.

9 *Corresponding author: Shuhao Qin: qinsh@gsau.edu.cn;

$10 \quad$ Tel.: +011861389313557

\section{Abstract}

Lectin receptor-like kinases (LecRLKs) are involved in response to diverse environmental stresses and pathogenic

microbes. A comprehensive acknowledgment of the family members in potato (Solanum tuberosum) genome is largely

limited until now. In total, 113 potato LecRLKs (StLecRLKs) were first identified, including 85 G-type, 26 L-type

and 2 C-type members. Based on phylogenetic analysis, StLecRLKs were sub-grouped into seven clades, analysis revealed the expansion of StLecRLKs occurred majorly through tandem duplication although the wholegenome duplication (WGD)/segmental duplication events were found. Cis-elements in the StLecRLKs promoter region responded mainly to signals of defense and stress, phytohormone, biotic or abiotic stress. Moreover, expressional investigations indicated that the family members of the clades L-type, G-I, G-IV and G-V were 
22 PGSC0003DMP400055136 and PGSC0003DMP400067047 were strongly induced in all treatments by both

23 Fusarium sulphureum (Fs) and Phytophthora infestans (Pi) inoculation. The present study provides valuable

24 information for LecRLKs gene family in potato genome, and establishes a foundation for further research into

25 the functional analysis.

26 Keywords Potato; Lectin receptor-like kinase (LecRLKs); gene duplication; expressional pattern; RNA-seq-based-

transcriptomics; fungal disease resistance 


\section{Introduction}

Unlike animals, plants lack the basic survival ability to escape from the danger of environmental fluctuations. However, receptor-like kinases (RLKs), as one of the largest receptors, allow the plants to communicate between cells and to interact with the environment [1]. RLKs also have an important role in response to biotic and abiotic stresses [2]. Typical RLKs comprise an extracellular ligand binding domain in $\mathrm{N}$ terminus and an intercellular cytoplasmic kinase domain in $\mathrm{C}$ terminus, and the two regions are connected via a transmembrane domain [3]. Based on sequence analysis of the variable extracellular domain and the kinase domain, a total of 747 RLKs have been categorized into 52 subfamilies [4]. However, Lectin receptor-like kinase (LecRLK) family was first described early in 1996 [5]. Previous study shows that there are a total of 75 and 173 LecRLKs in Arabidopsis and rice, respectively [3]. Expression of the LecRLK proteins have been reported in Lombardy poplar [6], Oryza sativa [7], Nicotiana benthamiana [8], Nicotiana tabacum [9], Nicotiana attenuata [10] Medicago truncatula [11] and Pisum sativum [12], although no LecRLK has been found in yeast and humans [11].

Depending on the variability of $\mathrm{N}$ terminal lectin domain, LecRLKs has been classified into three types: G-, L- and C-type [13]. G-type LecRLKs possess $\alpha$-D-mannose specific plant lectins. Many G-type LecRLKs also contain a cysteine-rich epidermal growth factor (EGF) domain and/or a plasminogen/apple/nematode (PAN) domain. However, EGF and PAN domains are only found in the G-type LecRLKs. The L-type LecRLKs contain a characteristic legume-lectin domain, which are similar with soluble lectin proteins found in leguminous plants [14]. The C-type LecRLKs are thought to be homologues of calcium-dependent lectin domain. Previous study has identified 32 G-type and 42 L-type LecRLKs in Arabidopsis and 100 G-type and 72 L-type members in rice. However, results showed that both Arabidopsis and rice have only one gene encoding C-type LecRLK protein [15]. 
LecRLKs have been reported to play a diverse role in plant multiple development stages, including seed

50 germination, senescence, responses to wounding and salinity [16,17]. Moreover, LecRLKs have been believed to be involved in tolerance to biotic and abiotic stresses, especially in pathogen attack [18-21], which is similar

to the role of RLKs reported by Nazarian-Firouzabadi et al. (2019) [2]. Transcript-level analyses revealed variable gene expression responses to diverse abiotic stresses, such as salt, drought, wounding and extreme temperatures $[3,16]$. The inhibition of LecRK-b2 can result in salt- and osmotic stress-insensitive root elongation [22]. The overexpression of Arabidopsis thaliana L-type lectin-like protein kinase 1 (AtLPK1) can enhance seed germination and cotyledon greening under high salinity conditions [23]. The overexpression of Glycine sojaLec RLK (GsSRK) and Pisum sativum LecRLK (PsLecRLK) can enhance salt tolerance [17,24]. The LecRLKs are also considered to be potential plant immune receptors, which play fundamental role in microbe- and plantderived molecular patterns associated with pathogen defense as the key pattern-recognition receptors $[19,21,25,26]$. Several LecRLKs played role in conferring resistance to rice against fungal pathogen [7] and bacterial pathogens [19].

Potato (Solanum tuberosum L.) is considered as one of the valuable food security crops in the word, with a global production of 375 million tons in 2018 [27]. The cultivated potato ranks fourth food crops planted and consumed worldwideafter rice, wheat and maize [28]. China, India, and Russia are principal potato producers [27]. However, because of the spread of pathogenic microorganisms, crop capacity and quality of potatoes produced by these countries are far behind those in developed countries. Date showed that the total annual potato loss due to bacteria/fungi and viruses worldwide is $14 \%$ and 7\%, respectively [29]. In Gansu province of China, late blight and dry rot, caused by Phytophthora infestans $(P i)$ and Fusarium sulphureum $(F s)$, respectively, result in huge losses in yield [30,31]. 
70 With the development of high-throughput sequencing technology, the transcriptome of potato and some 71 other plants have been published, which have laid a foundation for the identification of large gene families.

72 Besides Arabidopsis and rice, LecRLKs gene have been identified in different plants including Populus, soybean, shrub moss and Eucalyptus [32]. However, limited information is available about LecRLKs in potato.

In the current study, we firstly conducted a comprehensive bioinformatics analysis of the LecRLKs in potato genome. The evolutional characteristics were investigated through phylogenetic relationship, chromosomal distribution and gene duplication events. Moreover, based on the published expression data and quantitative realtime PCR (qRT-PCR), the expressional patterns of the family members were analyzed during potato against to biotic stresses, which provided a novel insight for the functional analysis of the LecRLK gene family in potato. 


\section{Methods}

\subsection{Annotation of LecRLKs}

The amino acid and nucleotide sequence data of Potato (Solanum tuberosum) v3.4, Arabidopsis thaliana Araport11, Rice (Oryza Sativa Japonica Group) IRGSP-1.0, Apple (Malus domestica) GDDH13 v1.1, and Tomato (Solanum lycopersicum) SL2.50 were downloaded from the Potato Genome Sequencing Consortium (PGSC) Public Data (http://solanaceae.plantbiology.msu.edu/pgsc download.shtml), The Arabidopsis Information Resource (TAIR) (https://www.arabidopsis.org), the Ensembl Genomes (http://ensemblgenomes.org/), the genome database for Rosaceae (GDR) (https://www.rosaceae.org/), and the Solanaceae Genomics Network (https://solgenomics.net/), respectively. Proteins contained kinase domain (PF00069) or Pkinase_Tyr (PF07714) in above five species were following the method described by Zhu et al. (2018) [33]. After that, sequences contained a N-terminal domain B_lectin (PF01453), Lectin_legB (PF00139) or Lectin_C (PF00059) were considered as LecRLKs. All of the candidate proteins were determined by using online software Pfam (http://pfam.xfam.org/) and SMART (http://smart.embl-heidelberg.de/) (E-value $<10^{-5}$ ) to ensure the presence of $\mathrm{N}$-terminal lectin domain, a transmembrane region and $\mathrm{C}$-terminal kinase domain in each putative member.

\subsection{Multiple sequence alignment, phylogenetic analysis, physical and chemical properties and subcellular}

\section{localization classification}

The sequences of all LecRLKs from six species were aligned using ClustalX 2.0 with default parameters [34] and a phylogenetic tree was constructed using the Neighbor-Joining (NJ) method in MEGA 7 [35]. The parameter partial deletion and P-distance Model were used with 1000 bootstrap replicates. The protein theoretical molecular weight (MV) and theoretical isoelectric point (pI) were predicted using ProtParam 
100 (https://web.expasy.org/protparam/)[36]. The subcellular localization of proteins was predicted using the WOLF

101 PSORT (https://www.genscript.com/wolf-psort.html) and CELLO v2.5: subcellular Localization predictor

102 (http://cello.life.nctu.edu.tw/).

103

104

105

106

107

108

109

110

111

112

113

114

115

116

117

118

119

120

2.3 Analysis of chromosomal distribution, gene duplication and Gene Ontology (GO) overview

The chromosomal positions of potato LecRLKs (StLecRLKs) were obtained from PGSC (http://solanaceae.plantbiology.msu.edu/pgsc_download.shtml) and the chromosomal distribution were visualized by using software MapDraw 2.1 [37]. Tandem duplicated genes are defined as those genes that (1) are $\leq 10$ genes apart, (2) belong to the same phylogenetic group, and are (3) within $100 \mathrm{~kb}$ [38]. The WholeGenome Duplication (WGD)/segmental duplication was calculated by MicroSyn software [39]. The projected GO annotation for the candidate StLecRLK genes was then analyzed for enrichment of GO terms using GOEast with default settings [40]. For cis-acting element analysis, genomic DNA sequences in the promoter region (-1500 bp) were downloaded from PGSC and then scanned for in the PlantCARE database (http://bioinformatics.psb.ugent.be/webtools/plantcare/html/) [41].

\subsection{Expression patterns of StLecRLKs in response to biotic infection}

To investigate the responses of StLecRLKs to biotic stress, expression data were obtained from the published studies, including potato infected with pathogenic bacteria Candidatus Liberibacter solanacearum (Cls) [42], Pectobacterium carotovorum subsp. brasiliense (Pcb) [43] and Ralstonia solanacearum (Rs) [44], fungi

Phytophthora infestans (Pi) [45], and Fusarium sulphureum (Fs) [46]. The expression pattern was extracted by the accession number of $S t$ LecRLKs. False Discovery Rate (FDR) $<0.05$ and $\mid \log 2$ (fold change) $\mid \geq 1$ were used as a threshold to judge the significance of the difference in gene expression. The heatmap was visualized by using software Multi experiment viewer software (MeV 4.9.0) (http://mev.tm4.org/). 


\subsection{Plant materials, treatment and gene expressional assay}

Potato cultivar Helan 15 (Favorita, Qingshenshu 2007001) were cultured in the greenhouse with $24^{\circ} \mathrm{C}$. After 5 weeks cultivation, leaf tissues were collected, sterilized with $75 \%$ ethanol and transferred into a petri dish with a diameter of $9 \mathrm{~cm}$. To maintain humidity, a wet filter paper was placed in each petri dish. $P i$ strain "HB09-162 (race 1.2.3. Zhendong, Huazhong Agricultural University. The pathogen was cultured at $19^{\circ} \mathrm{C}$ for two weeks. The conidia were collected and adjusted to a concentration as $7 \times 10^{4}$ sporangia per milliliter. Inoculation was performed following the method described by He et al. (2018) [46]. For the control, the conidia suspension was replaced with the same volume of sterilized water. Inoculated and control tissues were collected in triplicate at $1 \mathrm{~d}, 4 \mathrm{~d}, 6$ $\mathrm{d}$, and $8 \mathrm{~d}$ after inoculation and quick-frozen in liquid nitrogen and stored at $-80^{\circ} \mathrm{C}$.

The method of inoculation of $F s$ was performed as described by Li et al. (2009) [47]. Longshu No.7 (Guoshenshu 2009006), without physical injuries or visible infection, was used as the material for the inoculation for $1 \mathrm{~d}, 2 \mathrm{~d}, 3 \mathrm{~d}$ and $4 \mathrm{~d}$. Before treatment, tubers were surfaced-disinfected with $2 \%$ sodium hypochlorite for 3 min, and then rinsed with tap water and air-dried.

RNA isolation, reverse-transcription and quantitative real-time PCR (qRT-PCR) were performed following the method described by Zuo et al. (2018) [48]. Sequences of primers and actin genes could be found out in File S1. The relative expression levels of each gene were calculated based on the $2^{-\Delta \Delta C T}$ method [49].

The above samples at different treatment time were collected from three independent replicates. Each experimental unit consisted of three plants or tubers, three leaves per plant and each leaf or tuber with one inoculation site. The data were statistically analyzed using software Origin 8.0 and the significant differences were analyzed using the t-test $(* P<0.05 ; * * P<0.01)$. 


\section{3. Results}

\subsection{LecRLKs annotation and classification}

144 Based on domain screening, a total of 113 transcripts encoding LecRLKs were identified in potato, including 85

G-type, 26 L-type and 2 C-type members (File S2). For some transcripts, two or more transcripts had the same genome loci and corresponded with the same gene accession, but most of these showed the distinct sequences.

Therefore, we determined the number of LecRLKs as the transcript's numbers. With the same criteria, 80, 76,

139 and 167 LecRLKs were annotated in Tomato (Solanum lycopersicum), Arabidopsis, Rice (Oryza sativa) and

Apple (Malus domestica), respectively. From the published study, we also re-identified the LecRLKs in different

plant species, including Populus, Eucalyptus, Shrub, Soybean, Corn and Moss (Table 1; [32]). To ensure the

uniformity of the obtained data, domains of all sequences were determined based on the same criterion as above.

We excluded several proteins which did not contain typical domains of LecRLKs (File S2). Compared to Moss,

LecRLKs in all the detected plants. Over half of LecRLKs were classified as G-type except in Shrub,

Arabidopsis, Corn and Moss. In potato, tomato, apple and Populus, G-type LecRLKs accounted for more than

$70 \%$ of the LecRLKs, which suggested that the G-type LecRLKs were rapidly expanded in potato, tomato and

Populus than in the other detected plants.

To further investigate the classification and evolution relationships of LecRLKs, phylogenetic tree of three 
163 (Figure 1). Clades G-I, G- II, G-IV and G-V contained LecRLKs family members from all the tested plants,

164 while G-III only contained members from rice. Potato G-type LecRLKs were discovered from four clades except

165 G-III. There were 28, 3, 25 and 29 members in G-I, G- II, G-IV and G- V clade which accounted for 25.93\%,

$1667.14 \%, 25 \%$ and $26.61 \%$ in each subgroup respectively.

L-type LecRLKs was subdivided into eight clades (Figure 2). L-V had two Arabidopsis thaliana LecRLKs.

L-VI contained members from four investigated plant species except rice. Other subgroups contained members

from all five species. Potato LecRLKs located in six clades except L-I and L-V. C-type LecRLKs could be

divided into four subgroups (Supplementary Figure 1), in which subgroup IV contained members from potato

and tomato. For the other subgroups, each of these only contained members from one single plant species. Taken

together, these results indicated the distinctly evolutional process of LecRLKs in plants. The division of each

clade was supported by high bootstrap values.

3.2 StLecRLKs classification, domain organization, physicochemical properties and gene ontology (GO)

\section{analysis}

To further confirm the classification and evolutionary relationship of StLecRLKs, phylogenetic tree generated from the alignment result of the full-length amino acid sequences (Figure 3 and File S3). StLecRLKs could be divided into seven clades, including C-type, L-type, G-I, G-II, G-III, G-IV and G-V, which contained 2, 26, 28, $3,8,15$ and 31 members, respectively.

Domain organization was also investigated for each subgroup of StLecRLKs (File S2). Unique N-terminal domain was discovered from both L-type (Lectin_legB) and C-type members (Lectin_C). However, complex domain composition was found from G-type LecRLKs. Besides B_lectin domain, multiple additional N-terminal domains were detected, such as S_locus_glycop (PF00954), PAN_2 (Pfam accession number was not detected) 
and EGF (SM000181). Additionally, two intracellular domains, DUF3660 (PF12398) and DUF3403 (PF11883)

were observed from some members. Domain DUF3403 was in C-terminal of specific protein, but its function is still unknow. Above all, G-type StLecRLKs, being the largest group, showed a more complex evolutionary relationship and domain organization than the other types.

After determining the conserved domains of StLecRLKs, the physicochemical properties and subcellular localization of these proteins were also analyzed (File S4). The results showed that 113 StLecRLKs had an amino acid size ranging from 419 to 1504 aa, a molecular weight ranging from 47.07 to $169.51 \mathrm{kDa}$, and pI value ranging from 5.02 to 9.26. The subcellular localization of 113 StLecRLKs were predicted by the online software CELLO and PSORT. 87 StLecRLKs were located in the plasma membrane, two in the cytoplasm, one was in the extracytoplasmic surface and three in both plasma membrane and cytoplasm. Moreover, there were 20 StLecRLKs with inconsistent prediction results from the two software. Further, 15 and 20 GO terms were distributed into molecular function and biological process (File S5). Within biological process, a larger number of family members involved in "metabolism process", "cell recognition", and "protein modification process".

For the molecular function categories, genes were mainly associated with "kinase activity", "catalytic activity", "transferase activity" and "binding".

\subsection{Chromosomal distribution, gene duplication}

Tandem duplication mechanism represents a common role in the expansion of RLK family. To identify the gene clusters and to investigate such events, we scanned the gene ID and the corresponded protein ID of StLecRLKs based on PGSC database (File S2). For several accessions, unique gene accession was corresponded to two or more protein accessions. For instance, gene PGSC0003DMG400019237 encoded three proteins, including 
StLecRLKs was visualized on chromosome across 12 chromosomes (Figure 4). There were 15, 10, 9, 20, 11 and 7

members scattered on Chr 02, 03, 04, 07, 09 and 10, respectively. Additionally, 15 tandem duplicated gene clusters

(marked in red vertical lines) and five WGD/segmental duplicated gene pairs (marked in blue dotted lines) were found from StLecRLKs. The tandem duplicated gene clusters contained 47 StLecRLKsconsisting of 40 G-type (black) and

7 L-type (green). On the other hand, WGD/segmental duplicated gene pairs contained seven G-type and two L-type

StLecRLKs. Above results indicated that tandem duplications played important roles in the amplification of the

StLecRLKs gene family.

\subsection{Cis-elements involved in StLecRLKs}

In order to further explore the functions of the candidate StLecRLKs, a 1500 bp promoter region was extracted and PlantCARE was used to analyze the cis-elements related to stress challenge. A great number of cis-elements responsive to defense and stress, phytohormines (abscisic acid (ABA), methyl jasmonate (MeJA) and salicylic acid (SA)), biotic stressors (elicitor) and abiotic stressors (drought, low temperature and wounding) were identified in the promoter region of StLecRLKs (Figure 5 and File S6). Of these, the number of genes which had the cis-element involved in ABA-, MeJA- and SA-responsiveness were 57, 57 and 34, respectively. Most of the StLecRLKs belonged to the phylogenetic group G-I, G-V and L-type. Additionally, some StLecRLKs had elements responsive to abiotic stress, and several members possessed elements responsible for elicitor. The above results indicated that StLecRLKs had a potential role in response to signals from phytohormones and multiple stresses (Figure 5).

\subsection{Expression patterns of $S t$ LecRLKs in response to biotic stresses}

Based on the published transcriptomic datasets, the expressional patterns of StLecRLKs were investigated during potato infected with phytopathogenic bacteria [42-44] and fungi $[45,46]$. For the bacteria, expression changes of 
226

227

228

229

230

231

232

233

234

235

236

237

238

239

240

241

242

243

244

245

246

StLecRLKs were extracted after potato inoculated with $C l s, P c b$ and $R s$ (Figure 6). A total of 55 StLecRLKs were

differently expressed, including 15 members in G-I, 2 in G-III, 10 in G-IV, 17 in G-V and 11 in L-type, respectively.

Furthermore, $28 \mathrm{StLecRLKs}$ were detected to be up-regulated under at least one bacterial infection.

Additionally, expression patterns of StLecRLKs were extracted from published transcriptomic data of potato tuber [50] and leaf [45] inoculated with Pi (Figure 7). A total of 26 members, including nine members in G-I (32.14\%),

two in G-III (25.0\%), two in G-IV (13.33\%), five in G- V (16.13\%) and eight in L-type (30.77\%) were differentially expressed (Figure 7). PGSC0003DMP400030282 showed the same expressional pattern among resistant tuber and leaves. However, PGSC0003DMP400032146 and PGSC0003DMP400055136 in G-I and PGSC0003DMP400045382

in G-IV were detected to be down-regulated under bacteria attack while they were up-regulated under fungi infection.

PGSC0003DMP400067047 in G-IV was down-regulated under both bacteria and fungi infection (Figure 6 and Figure

7). Combined with the above results, a great number of G-I, G-V and L-type StLecRLKs were differentially expressed after potato tissue inoculated with both phytopathogenic fungi and bacteria, which indicated the potential roles of these members in potato disease resistance.

\subsection{Expression patterns of $S t$ LecRLKs during potato infected with $F s$ and $P i$}

To further validate the regulating roles of the candidate StLecRLKs to fungal disease resistance, the expression analysis of StLecRLKs during potato inoculated with Fs (Figure 8) and Pi (Figure 9) were detected. Two or three members in each phylogenetic group were selected, which were strongly affected under the biotic stress treatment

(Figure 6 and Figure 7). Finally, 16 differently expressed members were selected to perform qRT-PCT analysis (File S7). During Fs infection, 10 of the 16 detected $S t L e c R L K s$ were differentially expressed at least one time point (Figure 8 and File S7), and they were detected to be up-regulated after treatments. The expression of PGSC0003DMP400067047 was gradually upregulated during the experiment, and the expression level was 26.59

Peer) reviewing PDF | (2019:09:41729:3:1:NEW 7 May 2020) 
247 times that of the control group at $4 \mathrm{~d}$ treatment. When the leaves were inoculated with $P i, 12$ representative genes

248 were differentially expressed (Figure 9 and File S7). PGSC0003DMP400041968, PGSC0003DMP400049549 and

249 PGSC0003DMP400055136 were significantly induced at the later stage (T4), and the expressional levels were 12.33,

250430.80 and 34.08 times that of the control group. PGSC0003DMP400030282, PGSC0003DMP400001977 and

251 PGSC0003DMP400045383 were significantly induced when the leaves were treated for $6 \mathrm{~d}$ (T3). 
252

253

254

260

261

262

263

264

265

266

267

268

269

270

271

272

\section{Discussion}

LecRLKs are considered as important regulators for external stimuli, such as environmental stresses and pathogen attack [51]. Previous literatures reported the identification, evolution and function of LecRLKs in several plant species [3,32,52-55]. Currently, a comprehensive analysis of the LecRLK family in potato was carried out, and 113 LecRLK genes including 85 G-type, 26 L-type and 2 C-type members were identified and analyzed. Furthermore, investigations for classification, evolution, gene ontology, cis-elements and expression patterns revealed several important features of the family members.

\subsection{Evolution and expansion of StLecRLKs}

Besides the differences in genome size, expansion rate is one of the most important contributors to the copy numbers of plant LecRLKs [54]. Compared to Moss, total numbers of LecRLKs were drastically expanded in higher plants (Table 1), which mainly resulted from the expansion of the G-type LecRLKs members, followed by L-type. In potato, tomato, apple and Populus, about three-quarter of LecRLKs were identified as G-type members, although L-type LecRLKs were more expanded than G-type members in several species such as Shrub, Arabidopsis and Corn (Table 1; [3]). The results showed that different plant species had different numbers of LecRLKs, which was possibly related with the different growth condition and growth behaviors, or maybe with the plant life cycles and the mode of reproduction. Yang et al. (2016) also speculated that the difference in the number of G-type and L-type LecRLKs between Arabidopsis and Populus may be related with the fact that Arabidopsis is a self-fertile plant whereas Populus is an obligate outcrossing plant [32]. In our study, the number of LecRLK in Arabidopsis and rice is different from the previous researchers, which may be due to the perfection of genome sequence or the difference of identification standards (Table 1).

Phylogenetic analysis revealed the distinct evolutionary process in plant species (Figure 1, 2 and File S1).

Peer] reviewing PDF | (2019:09:41729:3:1:NEW 7 May 2020) 
273 The rice specific clade was found both in G-type (G-III) and L-type (L-I) LecRLKs. Therefore, we suggested the 274 distinct expanded characteristics between G-type and L-type LecRLKs in plant species. Compare to L-type LecRLKs

275 in potato, more G-type members were found, indicating the crucial roles of G-type LecRLKs in development and environmental response.

\subsection{Gene duplications and functional analysis}

Duplication can lead to functional divergence of genes, which is a universal phenomenon in plants [56].

LecRLKs duplication were mainly resulted from tandem duplication and segmental duplication/WGDs

$[32,38,57]$. In potato, 40 G-type $S t$ LecRLKs were involved in tandem duplication but only seven members in

WGD/segmental duplication (Figure 4). Similarly, there were more tandem duplicated L-type $S t$ LecRLKs than that in

segmental duplication/WGD (Figure 4). Hence, the tandem duplication might be the main reason for expansion of

both G-type and L-type StLecRLKs. Compared to RLKs involved in development, stress responsive RLKs showed a

higher number of tandemly duplicated genes. Based on the above conclusion, we deduced that $S t$ LecRLKs also took

important roles in potato responses to biotic and abiotic stress. Furtherly, we used GO method to analyze the molecular

function of these genes. The results showed that the enriched GO terms were kinase activity, catalytic activity,

transferase activity and binding (phosphate binding and carbohydrate binding) (Figure 5). Since chitin and $\beta-1,3$

glucans are components of the cell walls of some higher fungi, some plant lectins sharing chitin-binding domains can

recognize the signal then naturally these substrates will be hydrolysed and fungal growth will be disrupted [58]. This

type of lectin could inhibit the growth of several phytopathogenic and saprophytic chitin containing fungi in vitro [59].

Moreover, the cis-element prediction furtherly demonstrated that StLecRLKs have the potential role against multiple 
294

295

296

297

298

299

300

301

302

303

304

305

306

307

308

309

310

311

312

313

314

\subsection{Complex domain architecture and organization of G-type StLecRLKs}

Protein domains are some complex regions of a protein's structure, which often perform some specific function

[62]. Architecture and composition of domain of G-type StLecRLKs were observed (File S2). In addition to

B_lectin domain, most G-type members contained an S_locus_glycoprotein, EGF and PAN_2 or PAN-AP

domain, in which PAN domains are involved in interactions of protein-protein and protein-carbohydrate [63].

Furthermore, some G-type StLecRLKs also contained intracellular domain DUF3660 or DUF3403. Our result

was consistent with the findings in Populus although the function of the two domains was largely limited until now [32]. Nevertheless, the complex domain architecture suggested the diverse functions of G-type StLecRLKs.

\subsection{StLecRLKs in response to biotic stresses}

Increased investigations reported the crucial role of both G-type and L-type LecRLKs in plant immunity [55, 61,64]. Over-expression of LecRK-V (L-type) significantly enhanced the resistance of Yangmai158 to powdery mildew [65]. Similar studies have been done in rice and tomatoes. Pid2 (G-type LecRLK in rice) and NbLecRK

(L-type LecRLK in tomato) were shown to increase the resistance to fungal pathogen and Phytophthora, respectively [7,21]. In this study, we found a large number of StLecRLKs were differentially expressed during potato infected both bacterial and fungal pathogens (Figure 6 and 7). Most of these were distributed in clades G-I, G-V and L-type. Genes which contributed to plant resistance to a wide of pathogens could be classified as broad-spectrum resistant genes [66,67]. In Populus, the root-expressed $P t$ LecRLKs maybe have the potential function for perceiving signals from soil microbes [32]. Intriguingly, the expression of PGSC0003DMP400030282 was induced under signals from both phytopathogenic bacteria and fungi, which suggested that the crucial roles in broad-spectrum disease resistance of potato, while PGSC0003DMP400055136 and PGSC0003DMP400067047 showed distinct expression, indicating a different role in response to different 
315

316

317

318

320

321

322

323

324

325

326

327

328

329

330

331

332

333

334

335

336

337

pathogen signal (Figure 7, 8 and Figure 9).

\section{Conclusions}

In conclusion, a systematic study was carried out to identify and characterize the LecRLKs family genes in potato. The phylogenetic analysis showed the distinctly evolutionary processes of the family members in different plants. G-type StLecRLKs exhibited a rapid enlargement of family members, followed by L-type members. The expansion of both G-type and L-type StLecRLKs was mainly resulted from the tandem duplication. In addition, a large number StLecRLKs were differentially expressed in response to bacteria and fungi. Among these, PGSC0003DMP400055136 and PGSC0003DMP400067047 were strongly induced by both $F s$ and $P i$, which could be used as candidate genes for functional analysis of resistance. Our study provides insight into further research on the functions and mechanisms of this important RLK subfamily.

Author Contributions: ZWN and QSH conceived the study idea, executed its design and coordination. ZWN performed the experiments and wrote the manuscript. ZWN and CZJ collected, analyzed, and deposited the data. FYL, KYC, YXY, SMF, YK and LYH proofread the final draft. All authors have read and approved the manuscript. Authors declare that none of the funding bodies have any role in the design of the study and collection, analysis, and interpretation of data as well as in writing the manuscript.

Funding: This research was supported by Discipline construction fund project of Gansu Agricultural University (GAU-XKJS-2018-225), National Natural Science Foundation of China (31260311), Natural Science Foundation of Gansu Province (1606RJZA034) and China Postdoctoral Science Foundation (2012M512042 and 2014T70942), China Agriculture Research System (CARS-09-P14).

Conflicts of Interest: The authors declare no conflict of interest.

Acknowledgements: We would like to thank Prof. Xiong Xingyao (The Institute of Vegetables and Flowers Chinese Academy of Agricultural Sciences, Beijing, China) for friendly providing Helan 15 potatoes. We also would like to thank Dr. Bai Lijun and Xu Tong (Chengdu Life Baseline Technology Co., LTD, Chengdu, China; 
$338 \mathrm{http}: / / \mathrm{www}$. lifebaseline.com/) for technical assistance with RNA sequencing and bioinformatic analysis.

\section{Availability of data and materials}

340 The data supporting the results of this article are provided as additional files. All the data pertaining to the present

341 study has been included in the tables/figures of the manuscript and the raw data of RNA sequencing has been

342 deposited at the Sequence Read Archive (SRA) database of NCBI (SRS4823473). 
344

345

346

347

348

349

350

351

352

353

354

355

356

357

358

359

360

361

362

363

364

365

366

367

368

369

370

371

372

373

374

375

376

377

378

379

380

381

382

383

\section{References}

1. Li, X., Salman, A., Guo, C., Yu, J., Cao, S., Gao, X., Li, W., Li, H., Guo, Y. Identification and characterization of LRR-RLK family genes in potato reveal their involvement in peptide signaling of cell fate decisions and biotic/abiotic stress responses. Cells 2018, 7, 120.

2. Nazarian-Firouzabadi, F., Joshi, S., Xue, H., Kushalappa, A. C. Genome-wide in silico identification of LysM-RLK genes in potato (Solanum tuberosum L.). Mol. Biol. Rep. 2019, 46, 5005-5017.

3. Vaid, N., Pandey, P. K., Tuteja, N. Genome-wide analysis of lectin receptor-like kinase family from Arabidopsis and rice. Plant Mol. Biol. 2012, 80, 365-388.

4. Dezhsetan, S. Genome scanning for identification and mapping of receptor-like kinase (RLK) gene superfamily in Solanum tuberosum . Physiol. Mol. Biol. Plants 2017, 23, 755-765.

5. Herve, C., Dabos, P., Galaud, J. P., Rouge, P., Lescure, B. Characterization of an Arabidopsis thaliana Gene that defines a new class of putative plant receptor kinases with an extracellular Lectin-like domain. J. Mol. Biol. 1996, 258, 778-788.

6. Nishiguchi, M., Yoshida, K., Sumizono, T., Tazaki, K. A receptor-like protein kinase with a lectin-like domain from Lombardy poplar: gene expression in response to wounding and characterization of phosphorylation activity. Mol. Genet. Genomics, 2002, 267, 506-514.

7. Chen, X., Shang, J., Chen, D., Lei, C., Zou, Y., Zhai, W., Liu, G., Xu, J., Ling, Z., Cao, G., Ma, B., Wang, Y., Zhao, X., Li, S., Zhu, L. A B-lectin receptor kinase gene conferring rice blast resistance. Plant J. 2006, 46, 794-804.

8. Kanzaki, H., Saitoh, H., Takahashi, Y., Berberich, T., Ito, A., Kamoun, S., Terauchi, R. NbLRK1, a lectinlike receptor kinase protein of Nicotiana benthamiana, interacts with Phytophthora infestans INF1 elicitin and mediates INF1-induced cell death. Planta 2008, 228, 977-987.

9. Sasabe, M., Naito, K., Suenaga, H., Ikeda, T., Toyoda, K., Inagaki, Y., Shiraishi, T., Ichinose, Y. Elicitinresponsive lectin-like receptor kinase genes in BY-2 cells. DNA Seq. 2007,18, 152-159.

10. Gilardoni, P. A., Hettenhausen, C., Baldwin, I. T., Bonaventure, G. Nicotiana attenuata LECTIN RECEPTOR KINASE1 suppresses the insect-mediated inhibition of induced defense responses during manduca sexta herbivory. Plant Cell 2011, 23, 3512-3532.

11. Navarrogochicoa, M., Camut, S., Timmers, A. C., Niebel, A., Herve, C., Boutet, E., Bono, J. J., Imberty, A., Cullimore, J. V. Characterization of four lectin-like receptor kinases expressed in roots of medicago truncatula: structure, location, regulation of expression, and potential role in the symbiosis with sinorhizobium meliloti. Plant Physiol. 2003, 133, 1893-1910.

12. Joshi, A., Dang, H. Q., Vaid, N., Tuteja, N. Pea lectin receptor-like kinase promotes high salinity stress tolerance in bacteria and expresses in response to stress in planta. Glycoconj J. 2010, 27, 133-150.

13. Shiu, S., Bleecker, A. B. Plant receptor-like kinase gene family: diversity, function, and signaling. Sci. Signal. 2001,113, 567.

14. Herve, C., Serres, J., Dabos, P., Canut, H., Barre, A., Rouge, P., Lescure, B. Characterization of the Arabidopsis lecRK-a genes: members of a superfamily encoding putative receptors with an extracellular domain homologous to legume lectins. Plant Mol Biol.1999, 39, 671-682.

15. Cambi, A., Koopman, M., Figdor, C.G. How C-type lectins detect pathogens. Cell. Microbiol. 2005, 7, 481488.

Peer] reviewing PDF | (2019:09:41729:3:1:NEW 7 May 2020) 
16. Vaid, N., Macovei, A., Tuteja, N. Knights in action: lectin receptor-like kinases in plant development and stress responses. Mol. Plant 2013, 6, 1405-1418.

17. Vaid, N., Pandey, P., Srivastava, V. K. Pea lectin receptor-like kinase functions in salinity adaptation without yield penalty, by alleviating osmotic and ionic stresses and upregulating stress-responsive genes. Plant Mol. Biol. 2015, 88, 193-206.

18. Cheng, X., Wu, Y., Guo, J., Du, B., Chen, R., Zhu, L. L., He, G. A rice lectin receptor-like kinase that is involved in innate immune responses also contributes to seed germination. Plant J. 2013, 76, 687-698.

19. Singh, P., Zimmerli, L. Lectin receptor kinases in plant innate immunity. Front. Plant Sci. 2013, 4, 124.

20. Liu, Y., Wu, H., Chen, H., Liu, Y., He, J., Kang, H., Sun, Z., Pan, G., Wang, Q., Hu, J., Zhou, F., Zhou, K., Zheng, X., Ren, Y., Chen, L., Wang, Y., Zhao, Z., Lin, Q., Wu, F., Zhang, X., Guo., X., Cheng., X., Jiang, L.,Wu, C., Wang, H., Wan, J. A gene cluster encoding lectin receptor kinases confers broad-spectrum and durable insect resistance in rice. Nat. Biotechnol. 2015, 33, 301-305.

21. Wang, Y., Cordewener, J. H., America, A. H., Shan, W., Bouwmeester, K., Govers, F. Arabidopsis Lectin Receptor Kinases LecRK-IX.1 and LecRK-IX.2 are functional analogs in regulating phytophthora resistance and plant cell death. Mol. Plant-microbe In. 2015, 28, 1032-1048.

22. Deng, K., Wang, Q., Zeng, J., Guo, X., Zhao, X., Tang, D., Liu, X. A Lectin receptor kinase positively regulates $\mathrm{ABA}$ response during seed germination and is involved in salt and osmotic stress response. J. Plant Biol. 2009, 52, 493-500.

23. Huang, P., Ju, H., Min, J., Zhang, X., Kim, S., Yang, K., Kim, C. Overexpression of L-type lectin-like protein kinase 1 confers pathogen resistance and regulates salinity response in Arabidopsis thaliana. Plant Sci. 2013, 203, 98-106.

24. Sun, X., Yu, Q., Tang, L., Ji, W., Bai, X., Cai, H., Liu, X., Ding, X., Zhu, Y. GsSRK, a G-type lectin Sreceptor-like serine/threonine protein kinase, is a positive regulator of plant tolerance to salt stress. J. Plant Physiol. 2013, 170, 505-515.

25. Wu, Y., Zhou, J. Receptor-Like Kinases in plant innate immunity. J. Integr. Plant Biol. 2013, 55, 1271-1286.

26. Huang, P., Yeh, Y., Liu, A., Cheng, C., Zimmerli, L. The Arabidopsis LecRK-VI.2 associates with the pattern-recognition receptor FLS2 and primes Nicotiana benthamiana pattern-triggered immunity. Plant J. 2014, 79, 243-255.

27. Potato Statistical Yearbook. Available online: http://www.nationalpotatocouncil.org/files/8415/3315/3604/2018_NPC_Statistical_Yearbook.pdf (accessed on 20 November 2018). 2018.

28. FAOSTAT. Crops production. Rome, Italy. http://faostat.fao.org. 2011.

29. OERKE, E. Crop losses to pests. J. Agric. Sci. 2006, 144, 31-43.

30. He, S., Jin, X., Wei, Z., Zhang, T., Du, X., Luo, D. Isolation and identification of pathogens causing dry rot of potato tuber in dingxi prefecture of gansu province. J. Yunnan Agr. Univ. 2004, 19, 550-552.

31. Hui, N., Li, J., Li, Q., Wang, L., Li, J., Ma, Y., Zhou, T. Mating type of potato Phytophthora infestans of Gansu and their sensitivity to Metalagl. Gansu Agr. Sci. Technol. 2010.

32. Yang, Y., Labbe, J., Muchero, W., Yang, X., Jawdy, S. S., Kennedy, M., Johnson J., Sreedasyam A., Schmutz, J., Tuskan, G.A., Chen, J. Genome-wide analysis of lectin receptor-like kinases in Populus. BMC Genomics 2016, 17, 699.

33. Zhu, K., Wang, X., Liu, J., Tang, J., Cheng, Q., Chen, J., Cheng, Z. The grapevine kinome: annotation, 
classification and expression patterns in developmental processes and stress responses. Hortic. Res. 2018, 5, 19.

34. Larkin, M. A., Blackshields, G., Brown, N. P., Chenna, R., McGettigan, P. A., McWilliam, H., Valentin, F., Wallace, I. M., Wilm, A., Lopez, R., Thompson, J. D., Gibson, T. J., Higgins, D. G. Clustal W and Clustal X version 2.0. Bioinformatics 2007, 23, 2947-2948.

35. Kumar, S., Stecher, G., Tamura, K. MEGA7: Molecular evolutionary genetics analysis version 7.0 for bigger datasets. Mol. Biol. Evol. 2016, 33, 1870-1874.

36. Gasteiger, E., Hoogland, C., Gattiker, A., Duvaud, S., Wilkins, M. R., Appel, R. D., Bairoch A.; Protein Identification and Analysis Tools on the ExPASy Server; (In) John M. Walker (ed): The Proteomics Protocols Handbook, Humana Press. 2005, 571-607.

37. Liu, R. H., Meng, J. L. MapDraw:A microsoft excel macro for drawing genetic linkage maps based on given genetic linkage data. Hereditas 2003, 25, 317-321.

38. Hofberger, J. A., Nsibo, D. L., Govers, F., Bouwmeester, K., Schranz, M. E. A complex interplay of tandemand whole genome duplication drives expansion of the L-type lectin receptor kinase gene family in the Brassicaceae. Genome Biol. Evol. 2015,7, 720-734.

39. Cai, B., Yang, X., Tuskan, G. A., Cheng, Z. MicroSyn: A user friendly tool for detection of microsynteny in a gene family. BMC Bioinformatics 2011, 12, 79-79.

40. Zheng, Q., Wang, X. J. GOEAST: a web-based software toolkit for Gene Ontology enrichment analysis. Nucleic Acids Res. 2008, 36(Web Server issue):W358-W363.

41. Lescot, M., Déhais, P., Thijs, G., Marchal, K., Moreau, Y., Van de Peer, Y., Rouzé, P., Rombauts, S. PlantCARE, a database of plant cis-acting regulatory elements and a portal to tools for in silico analysis of promoter sequences. Nucleic Acids Res. 2002, 30:325-327.

42. Levy, J., Mendoza, A., Miller, J. C., Tamborindeguy, C., Pierson, E. A. Global gene expression in two potato cultivars in response to 'Candidatus Liberibacter solanacearum' infection. BMC Genomics 2017, 18, 960960.

43. Kwenda, S., Motlolometsi, T. V., Birch, P. R., Moleleki, L. N. RNA-seq profiling reveals defense responses in a tolerant potato cultivar to stem infection by Pectobacterium carotovorum ssp. brasiliense. Front. Plant Sci. 2016, 7, 1905.

44. Zuluaga, A. P., Sole, M., Lu, H., Gongoracastillo, E., Vaillancourt, B., Coll, N. S., Robin Buell, C., Valls, M. Transcriptome responses to Ralstonia solanacearum infection in the roots of the wild potato Solanum commersonii. BMC Genomics 2015, 16, 246-246.

45. Yogendra, K. N., Kushalappa, A. C. Integrated transcriptomics and metabolomics reveal induction of hierarchies of resistance genes in potato against late blight. Funct. Plant Biol. 2016, 43, 766-782.

46. He, Q., McLellan, H., Hughes, R. K., Boevink, P. C., Armstrong, M., Lu, Y., Banfield, M. J., Tian, Z., Birch, P. R. Phytophthora infestans effector SFI 3 targets potato UBK to suppress early immune transcriptional responses. New Phytol. 2018, doi:10.1111/nph.15635.

47. Li, Y., Bi, Y., Ge, Y., Sun, X. J., Wang, Y. Antifungal activity of sodium silicate on Fusarium sulphureum and its effect on dry rot of potato tubers. J. Food Sci. 2009, 74, 213-218.

48. Zuo, C., Zhang, W., Ma, Z., Chu, M., Mao, J., An, Z., Chen, B. Genome-wide identification and expression analysis of the CrRLK1L gene family in apple (Malus domestica). Plant Mol. Biol. Rep. 2018, 36, 844-857.

49. Livak, K. J., Schmittgen, T. D. Analysis of relative gene expression data using real-time quantitative PCR 
and the 2(-Delta Delta C(T)) Method. Methods 2001, 25, 402-408.

50. Gao, L., Tu, Z. J., Millett, B. P., Bradeen, J. M. Insights into organ-specific pathogen defense responses in plants: RNA-seq analysis of potato tuber-Phytophthora infestans interactions. BMC Genomics 2013, 14, 340340 .

51. Bellande, K., Bono, J.J., Savelli, B., Jamet, E., Canut, H. Plant lectins and lectin receptor-like kinases: how do they sense the outside?. Int. J. Mol. Sci. 2017, 18(6), 1164.

52. Zhao, W., Liu, Y., Zhou, J., Zhao, S., Zhang, X., Min, D. Genome-wide analysis of the lectin receptor-like kinase family in foxtail millet (Setaria italica L.). Plant Cell Tiss. Org. 2016, 127, 335-346.

53. Ma, N., Liu, C., Li, H., Wang, J., Zhang, B., Lin, J., Chang, Y. Genome-wide identification of lectin receptor kinases in pear: Functional characterization of the L-type LecRLK gene PbLRK138. Gene 2018, 661, 11-21.

54. Liu, P., Huang, Y., Shi, P., Yu, M., Xie, J., Xie, L. Duplication and diversification of lectin receptor-like kinases (LecRLK) genes in soybean. Scientific Rep. 2018, 8.

55. Zhao, T., Wang, J., Zhang, B., Hou, X. Genome-wide analysis of lectin receptor-like kinases in tomato (Solanum lycopersicum) and its association with the infection of tomato yellow leaf Curl Virus. Plant Mol. Biol. Rep. 2018, 36, 429-438.

56. Liu, P., Wan, J., Guo, Y., Ge, S., Rao, G. Adaptive evolution of the chrysanthemyl diphosphate synthase gene involved in irregular monoterpene metabolism. BMC Evol. Biol. 2012, 12, 214-214.

57. Shumayla, S., S. N., Pandey, A. K., Singh, K., Upadhyay, S. K. Molecular characterization and global expressioa analysis of lectin receptor kinases in bread wheat (Triticum aestivum). Plos one 2016, 11, e0153925.

58. Sahai, A. S., Manocha, M. S. Chitinases of fungi and plants: their involvement in morphogenesis and hostparasite interaction. FEMS Microbio. Rev. 1993, 11(4), 317-338.

59. Broekaert, W. F., Parijs, J.V., Leyns, F., Joos, H., Peumans, W.J. A chitin-binding lectin from stinging nettle rhizomes with antifungal properties. Science 1989, 245, 1100-1102.

60. Xin, Z., Wang, A., Yang, G., Gao, P., Zheng, Z. L. The Arabidopsis A4 subfamily of lectin receptor kinases negatively regulates abscisic acid response in seed germination. Plant Physiol. 2009, 149, 434-44.

61. Wang, Y., Weide, R., Govers, F., Bouwmeester, K. L-type lectin receptor kinases in Nicotiana benthamiana and tomato and their role in Phytophthora resistance. J. Exp. Bot. 2015, 66, 6731-43.

62. Buljan, M., Bateman, A. The evolution of protein domain families. Biochem. Soc. T. 2009, 37, 751-755.

63. Naithani, S., Chookajorn, T., Ripoll, D. R., Nasrallah, J. B. Structural modules for receptor dimerization in the S-locus receptor kinase extracellular domain. P. Natl. Acad. Sci. USA 2007, 104, 12211-12216.

64. Wang, Y., Bouwmeester, K., Beseh, P., Shan, W., Govers, F. Phenotypic analyses of Arabidopsis T-DNA insertion lines and expression profiling reveal that multiple L-type lectin receptor kinases are involved in plant immunity. Mol. Plant-microbe In. 2014, 27, 1390-1402.

65. Wang, Z., Cheng, J., Fan, A., Zhao, J., Yu, Z., Li, Y., ...Wang, X. LecRK-V, an L-type lectin receptor kinase in Haynaldia villosa, plays positive role in resistance to wheat powdery mildew. Plant Biotechnol. J. 2018, $16,50-62$.

66. Dangl, J.L., Jones, J.D. Plant pathogens and integrated defence responses to infection. Nature 2011, 411, $826-833$.

67. Xiao, S., Emerson, B. C., Ratanasut, K., Patrick, E., Oneill, C. M., Bancroft, I., Turner, J. , Origin and maintenance of a broad-spectrum disease resistance locus in Arabidopsis. Mol. Biol. Evol. 2004, 21, 1661- 
Figure 1

Phylogenetic analysis of G-type LecRLKs from five different plants.

The phylogenetic tree was generated from the alignment result of the full-length amino acid sequences by the neighbor-joining (NJ) method. All StLecRLKs members, together with homologues of Arabidopsis thaliana, apple (Malus domestica), rice (Oryza sativa) and tomato (Solanum lycopersicum), were classified into five distinct clades. LecRLK subgroups were shown in different colors.

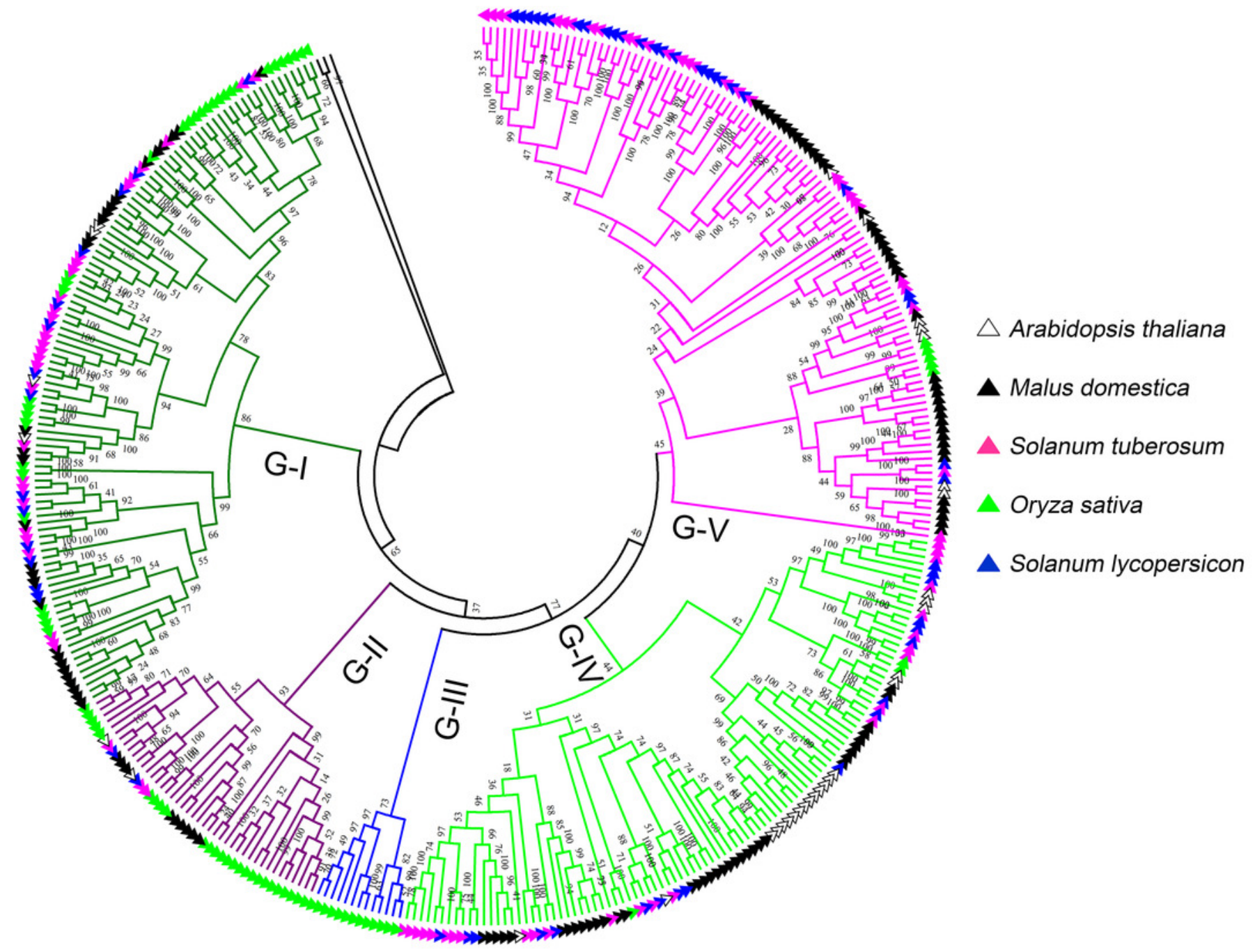


Figure 2

Phylogenetic analysis of L-type LecRLKs from five different plants.

The phylogenetic tree was generated from the alignment result of the full-length amino acid sequences by the neighbor-joining (NJ) method. All StLecRLKs members, together with homologues of Arabidopsis thaliana, apple (Malus domestica), rice (Oryza sativa) and tomato (Solanum lycopersicum), were classified into eight distinct clades. LecRLK subgroups were shown in different colors.

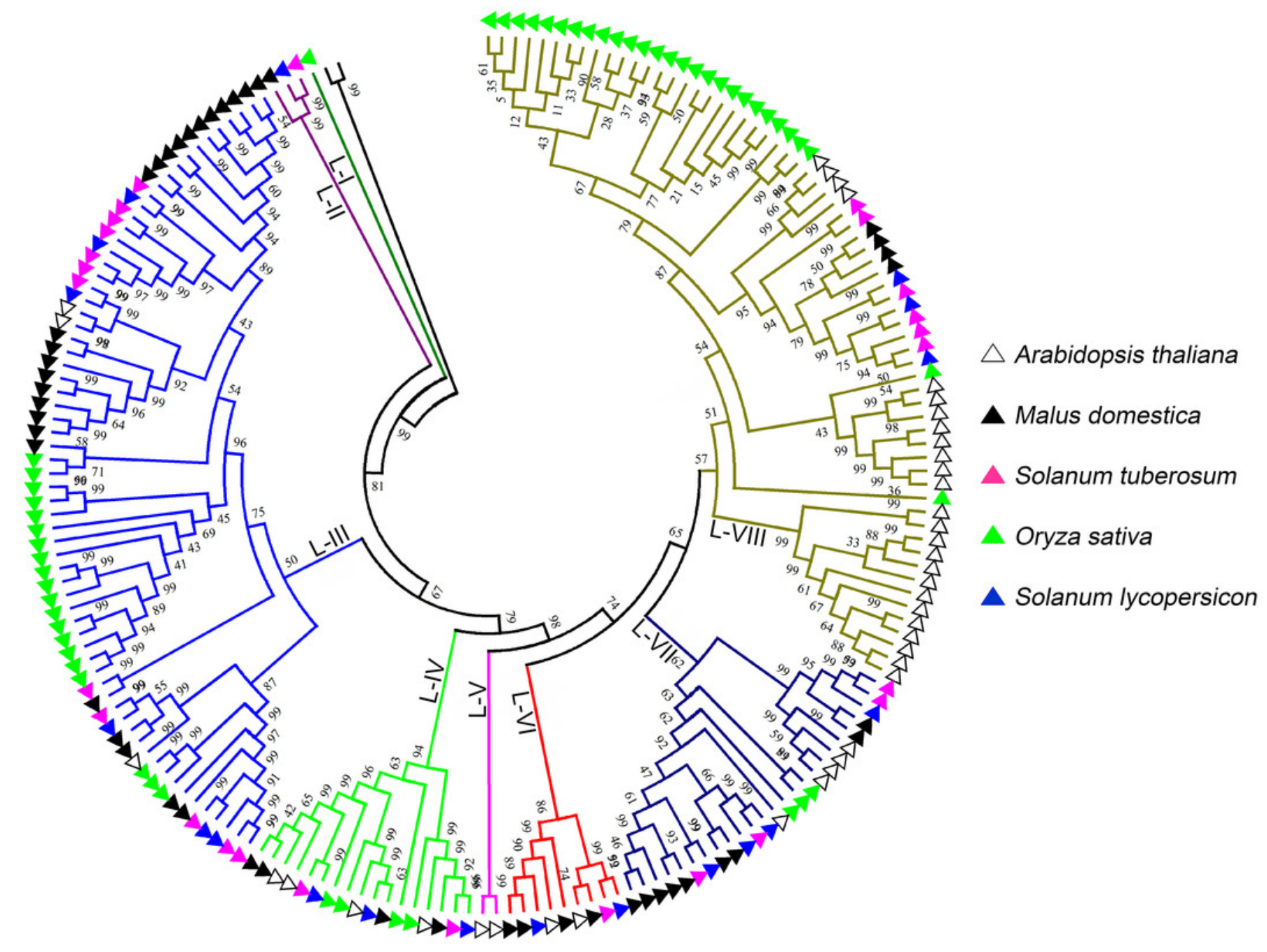


Figure 3

Phylogenetic analysis of LecRLKs in potato (Solanum tuberosum).

The phylogenetic trees were constructed using MEGA 7 software. The numbers were bootstrap values based on 1000 iterations. Note that three different types of StLecRLKs were categorized clearly in seven clades. Different color was used to distinguish different clades. 


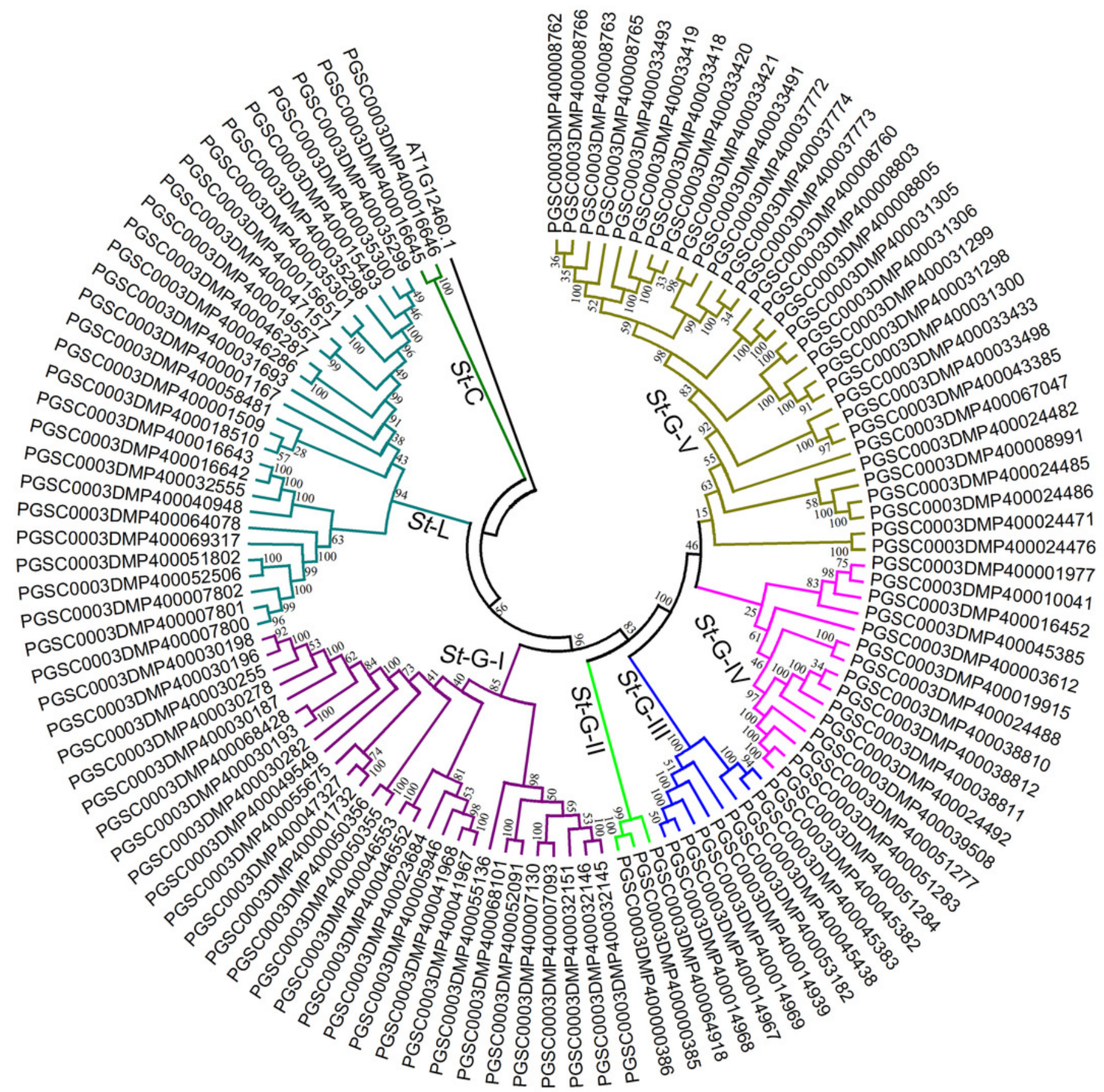

Peer] reviewing PDF | (2019:09:41729:3:1:NEW 7 May 2020) 
Figure 4

Distribution of LecRLKs genes on potato (Solanum tuberosum) chromosomes.

Graphical representation of physical locations for each LecRLK on chromosomes. Tandem duplicated genes on the particular chromosome were depicted by red vertical lines.

Chromosomal distances were given in $\mathrm{Mb}$.
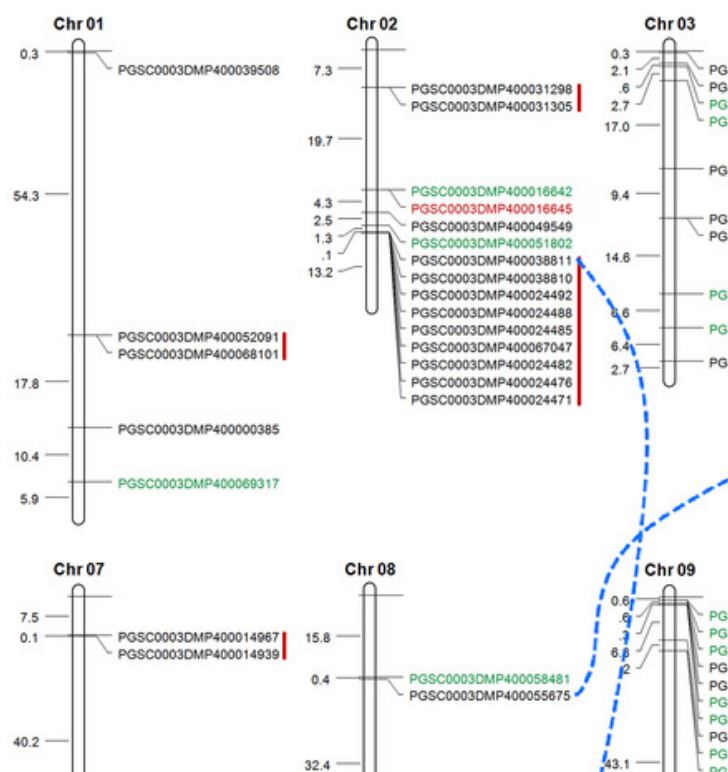

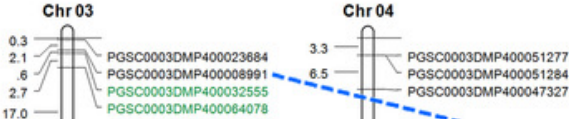

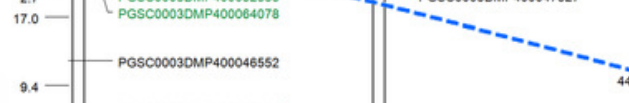

$$
\text { : }
$$
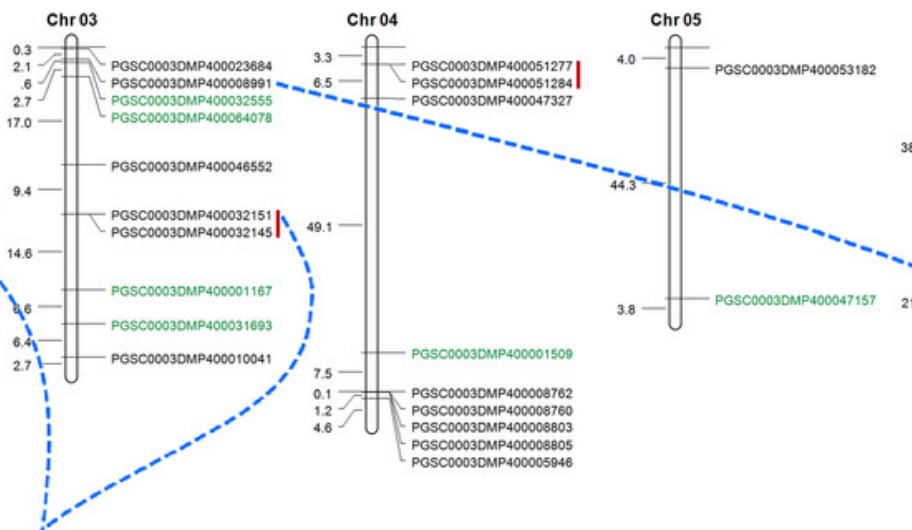

Chr 06
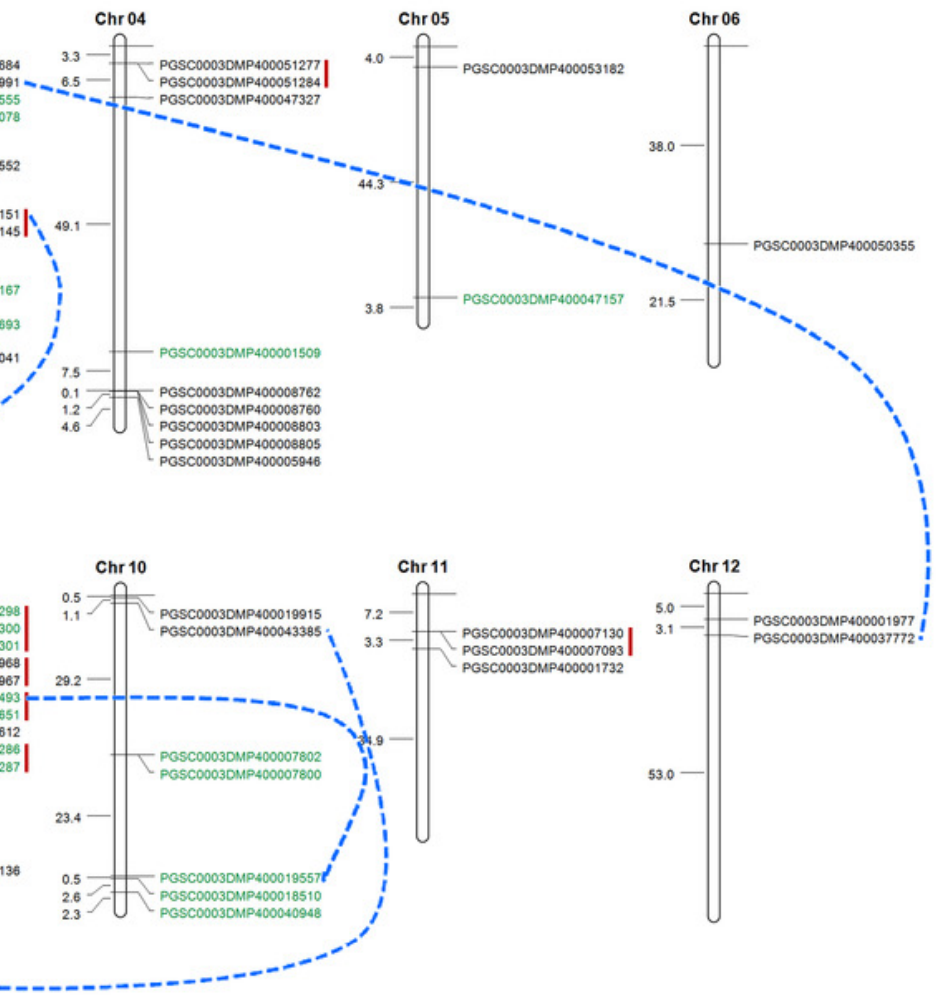


\section{Figure 5}

Cis-element detection in the promoter region of StLecRLK.

Signals of cis-element were responsive for stress challenge, including deference and stress, elicitor, low-temperature, drought, wounding and hormone (SA, ABA and MeJA). Different color was used to distinguish different clades.

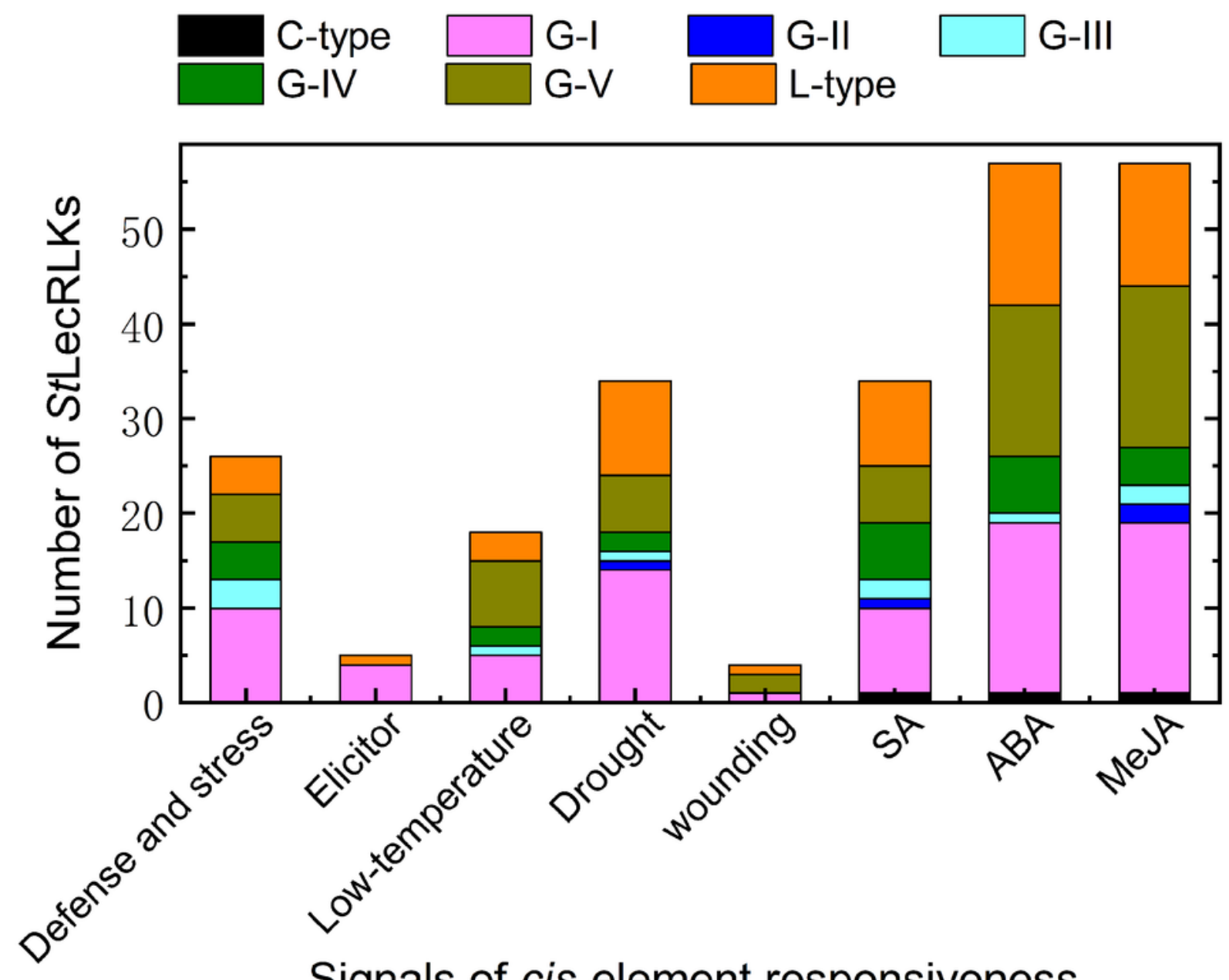

Signals of cis-element responsiveness 


\section{Figure 6}

Expression patterns of StLecRLKs in response to phytopathogenic bacteria.

The heatmap showed the differentially expressed genes of Waneta potato infected with Candidatus Liberibacter solanacearum $(\mathrm{Cl} / \mathrm{s})$, Solanum tuberosum $\mathrm{cv}$. Valor compared with $\mathrm{S}$. tuberosum Cv. BP1 at each time-point inoculated with Pectobacterium carotovorum ssp. brasiliense $(P c b)$, and differentially-expressed genes in inoculated potato roots with Ralstonia solanacearum (Rs). The classification of genes in phylogenetic trees were listed next to the gene name. Yellow indicated genes that were up-regulated, blue indicated genes that were down-regulated, and white indicated genes that were not changed significantly. 
$\begin{array}{lll}-5.0 & 0.0 & 5.0\end{array}$
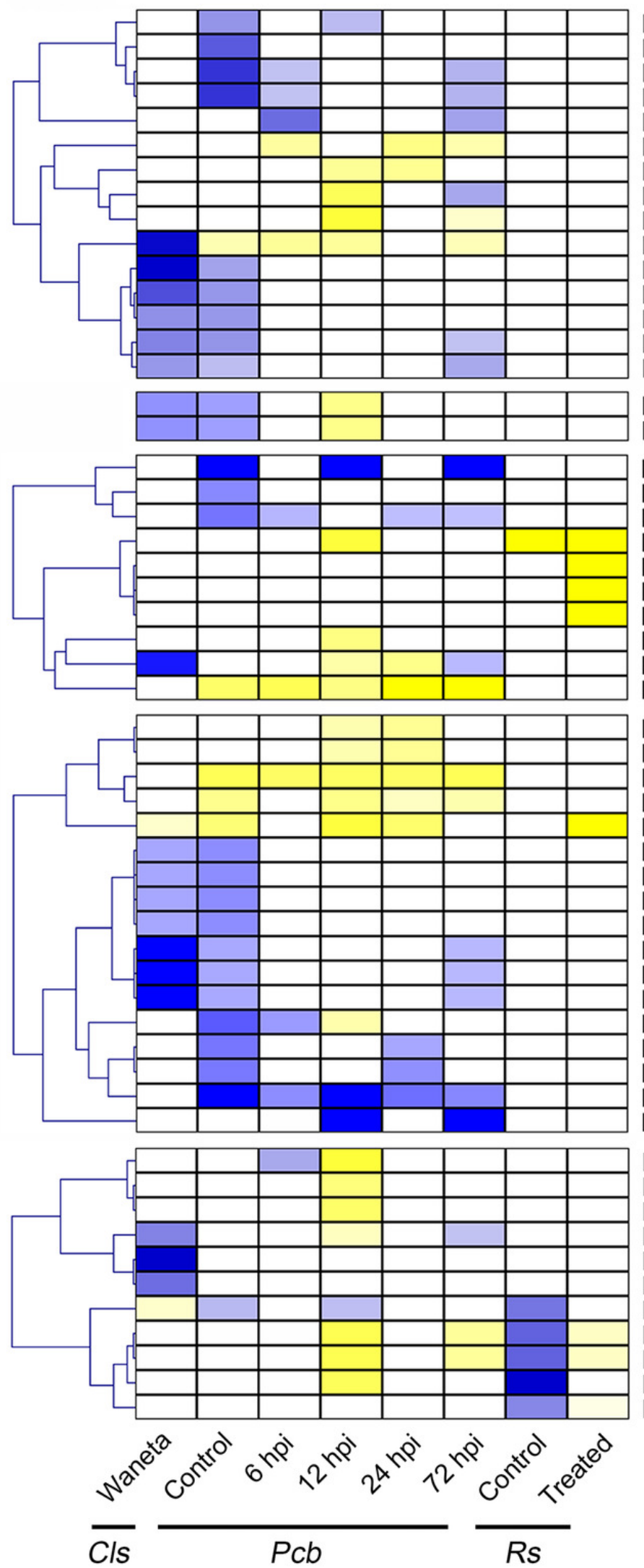

PGSC0003DMP400030196 PGSC0003DMP400030255 PGSC0003DMP400032145 PGSC0003DMP400032146 PGSC0003DMP400049549 PGSC0003DMP400030187 PGSC0003DMP400005946 PGSC0003DMP400050356 PGSC0003DMP400052091 PGSC0003DMP400030282 PGSC0003DMP400007093 PGSC0003DMP400007130 PGSC0003DMP400041968 PGSC0003DMP400032151 PGSC0003DMP400055136 PGSC0003DMP400045382 PGSC0003DMP400045383 PGSC0003DMP400003612 PGSC0003DMP400010041 PGSC0003DMP400024492 PGSC0003DMP400019915 PGSC0003DMP400038810 PGSC0003DMP400038811 PGSC0003DMP400038812 PGSC0003DMP400001977 PGSC0003DMP400024488 PGSC0003DMP400039508

PGSC0003DMP400031305 PGSC0003DMP400031306 PGSC0003DMP400033421 PGSC0003DMP400033498 PGSC0003DMP400043385 PGSC0003DMP400008763 PGSC0003DMP400008762 PGSC0003DMP400008765 PGSC0003DMP400008766 PGSC0003DMP400031298 PGSC0003DMP400031299 PGSC0003DMP400031300 PGSC0003DMP400024471 PGSC0003DMP400008805 PGSC0003DMP400024482 PGSC0003DMP400033493 PGSC0003DMP400067047 PGSC0003DMP400015651 PGSC0003DMP400035300 PGSC0003DMP400046287 PGSC0003DMP400031693 PGSC0003DMP400019557 PGSC0003DMP400052506 PGSC0003DMP400001167 PGSC0003DMP400007800 PGSC0003DMP400007801 PGSC0003DMP400015493 PGSC0003DMP400064078
St-G-I

St-G-III

\section{St-G-IV}

St-G-V 


\section{Figure 7}

Expression patterns of StLecRLKs in response to Phytophthora infestans (Pi).

The heatmap of differentially expressed genes between F06025 (derived from AWN86514-2 $\times$ N06993-13 cross) and F06037 (derived from frontier russet $\times$ N1503-19 cross) genotypes, RB (tubers of transgenic 'Russet Burbank' line SP2211 potato) and WT (tubers of nontransformed 'Russet Burbank' (WT) potato) inoculated with Pi. The classification of genes in phylogenetic trees were listed next to the gene name. Bins in yellow were significantly upregulated; bins in blue were significantly down-regulated and bins in white did not change significantly. 


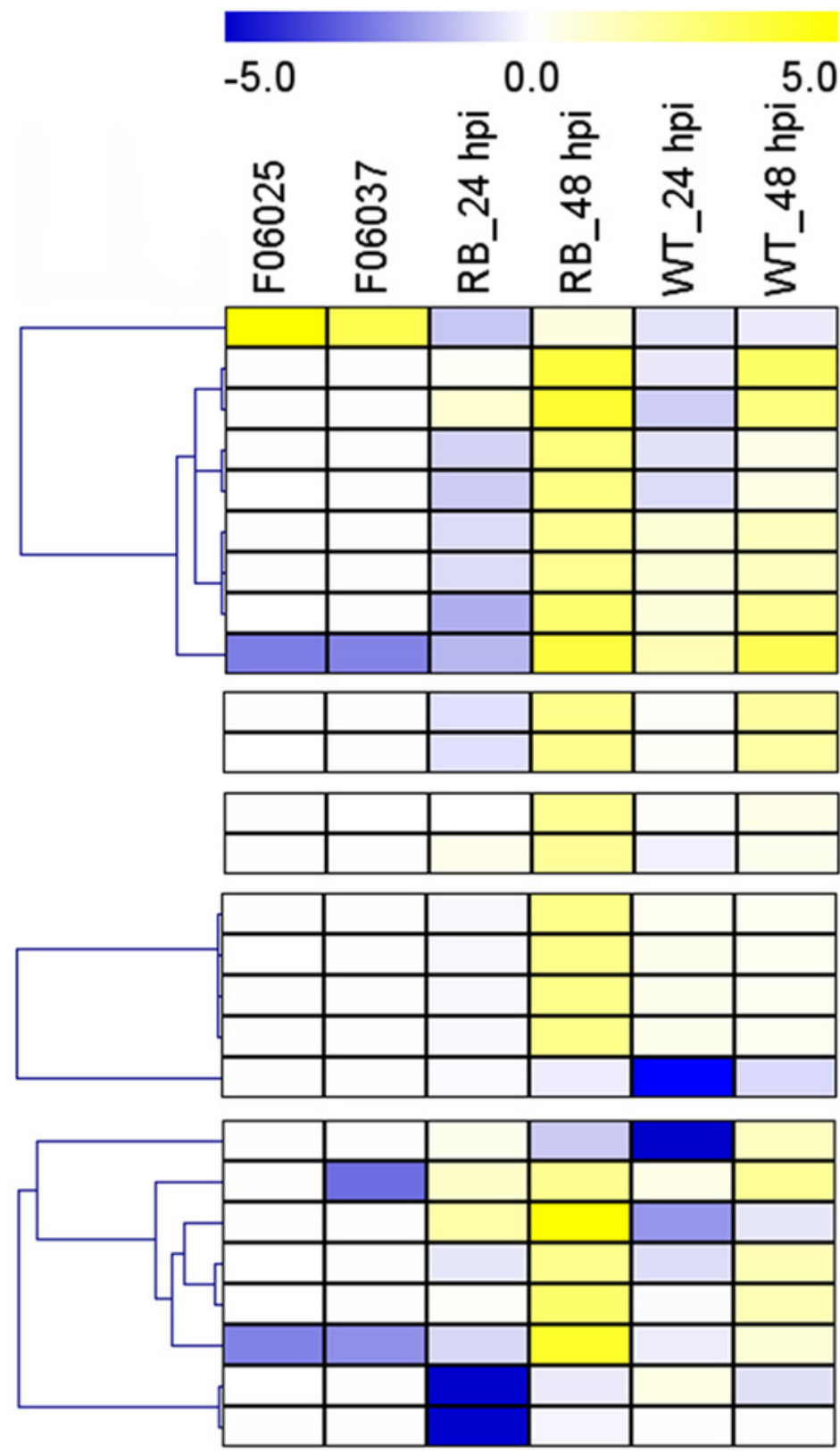

PGSC0003DMP400030282 PGSC0003DMP400007093 PGSC0003DMP400007130 PGSC0003DMP400041967 PGSC0003DMP400041968 PGSC0003DMP400032145 PGSC0003DMP400032146 PGSC0003DMP400049549 PGSC0003DMP400055136 PGSC0003DMP400045382 PGSC0003DMP400045383

St-G-III PGSC0003DMP400001977 PGSC0003DMP400019915 St-G-IV PGSC0003DMP400008763 PGSC0003DMP400008765 PGSC0003DMP400008762 PGSC0003DMP400008766 St-G-V PGSC0003DMP400067047 PGSC0003DMP400046287 PGSC0003DMP400040948 PGSC0003DMP400015651 PGSC0003DMP400007802 PGSC0003DMP400035301 St-L PGSC0003DMP400052506 PGSC0003DMP400001509 PGSC0003DMP400069317

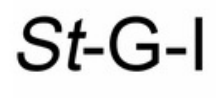


Figure 8

Expressions of 10 StLecRLKs during potato inoculated with Fusarium sulphureum (Fs).

(A-H) represent eight G-type LecRLK genes, in which A-G belonged to $\mathrm{G}-\mathrm{I}$ clade and $\mathrm{H}$ was in G-III clade; (I-J) two L-type LecRLK genes. Samples at 1-, 2-, 3- and 4-days post Fs inoculation were collected. The qRT-PCR data presented here were from three independent biological replicates and error bars indicated the standard deviation (SD). The asterisks indicated a significant difference as compared with the group inoculated with sterilized water at each time point $(* p<0.05$ and $* * p<0.01)$.

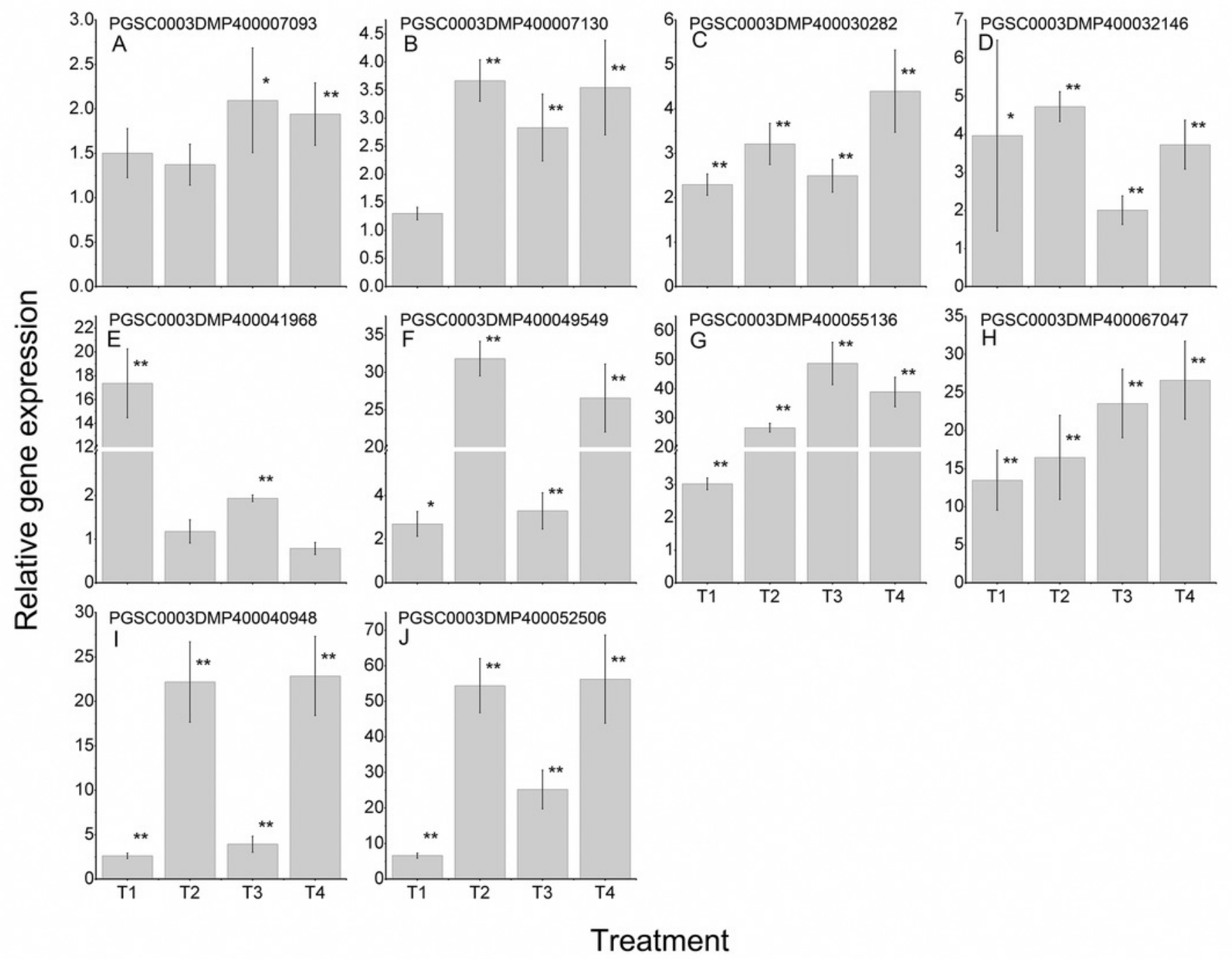


Figure 9

Expressions of 12 StLecRLKs during potato inoculated with Phytophthora infestans (Pi).

(A-J) represent 10 G-type LecRLK genes, in which A-F belonged to G-I clade, G was in G-II clade and H-J were in G-III clade; (K-L) two L-type LecRLK genes. Samples at 1-, 2-, 3- and 4days post $P i$ inoculation were collected. The qRT-PCR data presented here were from three independent biological replicates and error bars indicated the standard deviation (SD). The asterisks indicated a significant difference as compared with the group inoculated with sterilized water at each time point $\left(* p<0.05\right.$ and $\left.{ }^{* *} p<0.01\right)$.
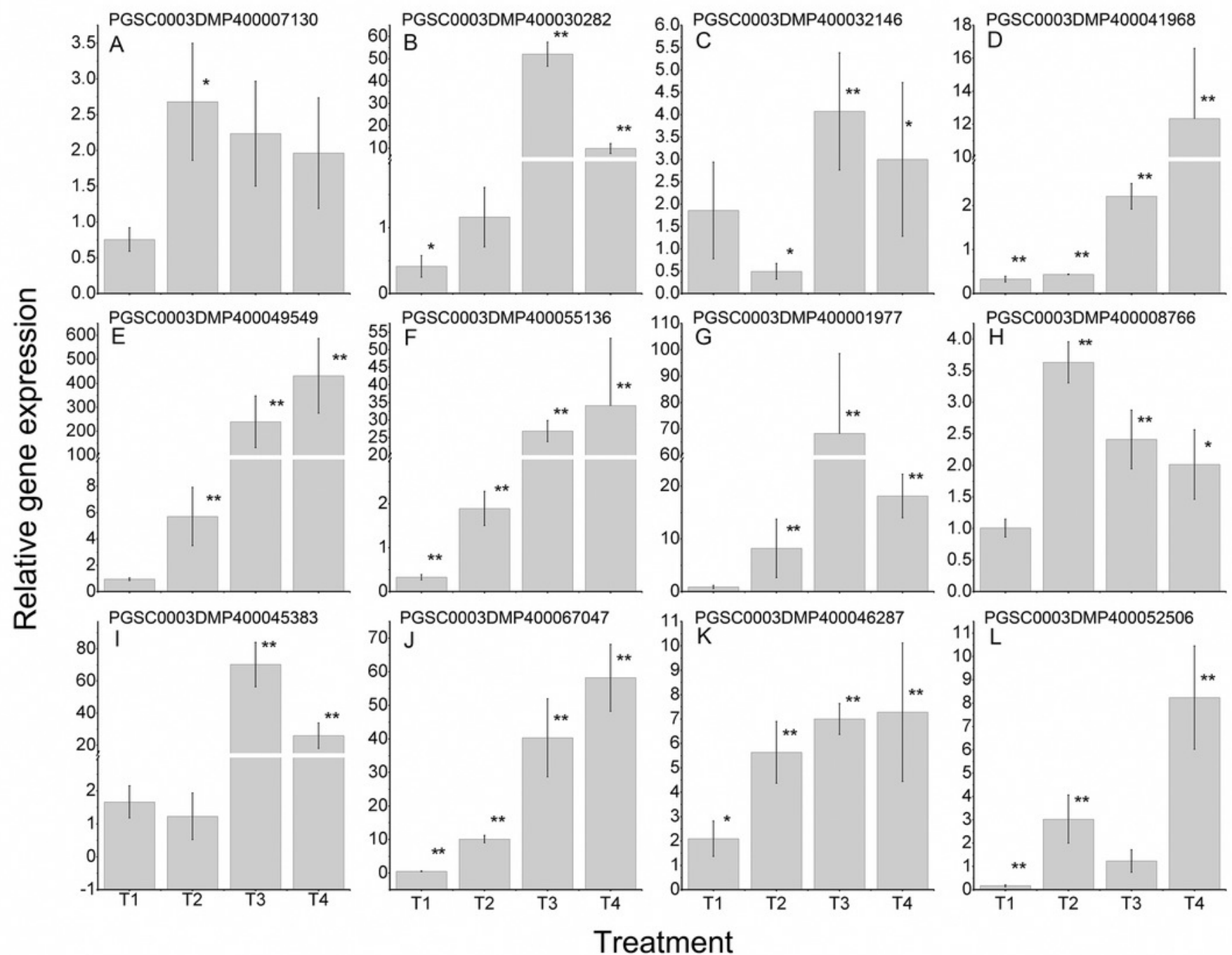

Treatment 


\section{Table 1 (on next page)}

The number of G-type, L-type and C-type LecRLKs in 11 plant species. 


\begin{tabular}{lllll}
\hline \multirow{2}{*}{ Plant species } & \multicolumn{3}{c}{ Sub-group } & Total \\
\cline { 2 - 5 } & G-type & L-type & C-type & 113 \\
\hline Solanum tuberosum & $85(75.22 \%)$ & $26(23.01 \%)$ & $2(1.77 \%)$ & 80 \\
Solanum lycopersicon & $60(75 \%)$ & $19(23.75 \%)$ & $1(1.25 \%)$ & 166 \\
Malus domestica & $118(71.08 \%)$ & $46(27.71 \%)$ & $2(1.2 \%)$ & $160)$ \\
Populus & $180(77.92 \%)$ & $50(21.65 \%)$ & $1(0.43 \%)$ & 231 \\
Eucalyptus & $118(59.6 \%)$ & $79(39.9 \%)$ & $1(0.51 \%)$ & 198 \\
Shrub & $25(44.64 \%)$ & $30(53.57 \%)$ & $1(1.79 \%)$ & 56 \\
Soybean & $123(65.08 \%)$ & $64(33.86 \%)$ & $2(1.06 \%)$ & 189 \\
Arabidopsis thaliana & $34(44.74 \%)$ & $41(53.94 \%)$ & $1(1.32 \%)$ & 76 \\
Oryza sativa & $80(57.55 \%)$ & $57(41.01 \%)$ & $2(1.44 \%)$ & 139 \\
Corn & $46(48.42)$ & $48(50.53 \%)$ & $1(1.05 \%)$ & 95 \\
Moss & $2(40 \%)$ & $1(20 \%)$ & $2(40 \%)$ & 5 \\
\hline
\end{tabular}

1 